\title{
FIXAÇÃO OCCÍPITO-CERVICAL "INSIDE-OUTSIDE"
}

\section{Relato técnico}

\author{
Igor de Castro', José Alberto Landeiro
}

RESUMO - O entendimento sobre a biomecânica do complexo occípito-atlanto-axial explica a instabilidade progressiva após a descompressão anterior da junção craniocervical.O propósito do presente relato é descrever a técnica de fixação occípito-cervical tipo "inside-outside" proposta por Pait et al., e os resultados em dois pacientes operados pela via transoral para remoção da apófise odontóide. A técnica utiliza uma haste de titânio moldada para adaptar-se à curvatura occipital e colocada lateralmente até a coluna cervical; a haste é fixada no osso occipital por meio de parafuso cuja parte achatada é colocada no espaço epidural. Na coluna cervical, a haste é fixada por parafusos que são introduzidos no quadrante súpero-lateral das massas articulares. No axis, o parafuso é introduzido na "pars interarticulares" podendo terminar no próprio corpo desta vértebra ou na massa articular do atlas. Esta técnica revelou-se segura e de fácil aplicabilidade.

PALAVRAS-CHAVE: fixação occípito-cervical, odontoidectomia, acesso transoral.

\section{Inside-outside occipito-cervical fixation: technical report}

ABSTRACT - The clinical knowledgement of biomechanics of atlantoaxial complex have been proved that progressive instability has a mandatory occurrence after anterior decompression of the craniocervical junction. We report the occípitocervical fixation so called inside-outside technique, originally described by Pait et al. appliedin in two patients whom underwent odontoidectomy. The occipitalcervical fixation technique consist in the use of a titanium rod bended according with occipital cervical angle placed and fixed laterally over the cervical spine. The rod is fixed to the occipital bone by mean of placement a screw which flat portion is positioned onto the epidural space. In the cervical spine the rod is attached to transarticular screws placed at the superolateral quadrant of the articular mass. In the axis the screw is introduced through the pars interarticularis finishing at the axis body or the lateral mass of the atlas. This technique proved to be safe and easily applied in the patients whom underwent this surgical procedure.

KEY WORDS: occípitocevical fixation, odontoidectomy, transoral approach.

O tratamento da instabilidade do complexo articular do osso occipital, atlas e axis é mais difícil do que o restante do segmento cervical. Técnicas de fixação occípito-cervical com órtese externa, enxertos ósseos, cabos e fios para fixação do enxerto, metilmetacrilato, substâncias osteoindutoras e, finalmente, o uso de instrumentais mais complexos como hastes, arames, placas e parafuso têm sido empregadas ${ }^{1-8}$. Meios de fixação como hastes, placas e parafusos promovem a estabilização imediata garantindo a segurança do paciente e a movimentação precoce. A instabilidade ocasionada pela descompressão por via anterior em lesões como invaginação basilar, artrite reumatóide e tumores ocorre em grande número de pacientes, exigindo que englobe o côndilo occipital (CO) e a primeira e segunda vértebras cervicais (C1 e C2).
O presente relato descreve a técnica proposta por Pait et al. ${ }^{1}$, de fixação occípito-cervical pelo método "inside-outside" com material especialmente designado para esta finalidade, ilustrada pelo relato de dois casos.

\section{TÉCNICA}

Os pacientes foram submetidos no pré-operatório a ressonância magnética (RM), tomografia computadorizada de crânio (TC) e da junção craniocervical (JCC) com reconstrução tridimensional para estudo da espessura do osso occipital, da integridade das vértebras cervicais, da espessura dos pedículos e do diâmetro dos forames vertebrais.

Os pacientes são operados em decúbito ventral, sob anestesia geral, com tração cervical em torno de $2 \mathrm{~kg}$ e a cabeça posicionada em suporte tipo ferradura coberta com espuma de silicone. $O$ pescoço é colocado em posição neutra e o arco em C posicionado para obter-se imagens late-

\footnotetext{
${ }^{1}$ Assistente do Serviço de Neurocirurgia do Hospital de Força Aérea do Galeão (HFAG) Rio de Janeiro RJ, Brasil; ${ }^{2}$ Chefe do Serviço de Neurocirurgia do HFAG e Professor Adjunto da Disciplina de Neurocirurgia da Faculdade de Medicina da Universidade Federal Fluminense, Niterói RJ, Brasil.
}

Recebido 19 Julho 2002, recebido na forma final 14 Setembro 2002. Aceito 21 Outubro 2002. 


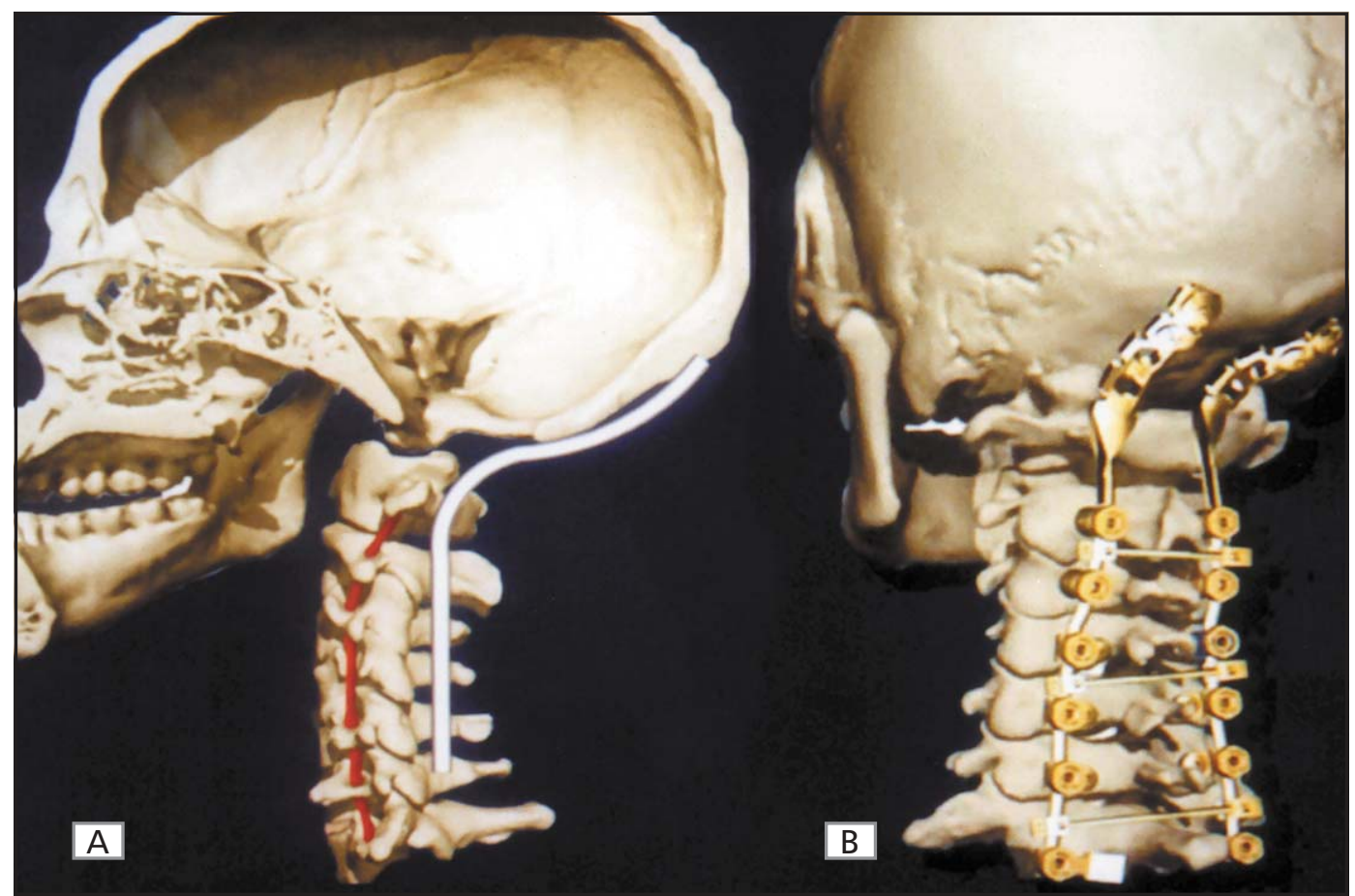

Fig 1. Sistema "inside-outside" de fixação occipito-cervical: esquema de colocação dos parafusos e das hastes. Observa-se que os parafusos são implantados a partir da pars articular de C2.

rais da JCC. Realizou-se incisão mediana que extende-se do ínion até C5. São expostos a escama occipital, o forame magno, C1 e C2 e no mínimo dois segmentos abaixo.

Os implantes utilizados são de titânio, compatíveis com RM e ajustáveis à curvatura occipital e transição craniocervical. Inicialmente, um modelo da haste é moldado à curvatura e colocado sobre o occipital e as massas articulares, sendo utilizado como ponto de marcação para o futuro alinhamento vertical dos parafusos tanto no occipital quanto nas massas articulares. Segue-se a trepanação na linha média da convexidade occipital para colocação da parte achatada do parafuso. O próximo passo é o posicionamento lateral do parafuso conseguido através de um trajeto no osso com a serra do craniótomo. Procede-se o posicionamento definitivo das hastes e o ancoramento das mesmas com os parafusos colocados nas massas articulares a partir de C2 . Os parafusos são implantados no quadrante súpero-lateral e dirigidos perpendicularmente em sentido proximal e voltado lateralmente com angulação de $20^{\circ}$. Após controle por imagens e verificação da correta posição dos parafusos é feito o aperto final (Fig 1). Fragmentos ósseos removidos da crista ilíaca são interpostos entre as hastes sobre os arcos das vértebras envolvidas, após brocagem da superfície óssea. Os pacientes são encaminhados à unidade de terapia intensiva com tubo orotraqueal e orogástrico. Esta técnica será demonstrada em dois pacientes.

O primeiro caso tratava-se de mulher de 36 anos, professora de educação física. Apresentava-se com diminuição da força no dimídeo esquerdo do corpo, perda do equilíbrio e dormência na língua à esquerda. Relatava dois episódios de perda súbita da consciência com pronta recu- peração, após aulas de educação física. A RM e TC-3D da JCC evidenciaram invaginação basilar com compressão anterior da transição bulbomedular. Foi submetida a odontoidectomia por via transoral com preservação parcial do arco anterior de C1. Um ano após a cirurgia começou a queixar-se de cervicalgia intensa, desencadeada por movimento do pescoço. Ao exame clínico constatou-se torcicolo espasmódico e as imagens obtidos por TC revelaram instabilidade do segmento CO C1 C2. Submetida a fixação occípitocervical com sistema "inside-outside" (Sistema Occifix - G.M Reis, Campinas, SP) com resolução dos sintomas (Fig 2).

$\mathrm{O}$ segundo paciente era uma mulher de 45 anos. Apresentava-se com tetraparesia espástica e as imagens de RM e TC-3D da JCC mostravam invaginação basilar, deslocamento posterior da odontóide com aumento do intervalo atlanto-odontóideo e consequente compressão ventral da junção bulbomedular. Foi em outro serviço submetida a craniectomia occipital e laminectomia de C1, sem melhora dos sintomas. Foi submetida a remoção por via transoral da apófise odontóide, do arco de C1 e do bordo inferior do clivo. No mesmo ato anestésico, foi colocada em decúbito ventral, sob tração cervical, e implantado o sistema de fixação occípito-cervical tipo "inside-outside", seguido da colocação de enxerto ósseo(fig.3). A paciente que se deslocava em cadeira de rodas, apresentou melhora progressiva da força dos membros inferiores e no último controle médico já se mantinha de pé com auxílio de suporte de braço.

\section{DISCUSSÃO}

A instabilidade craniocervical está associada com 


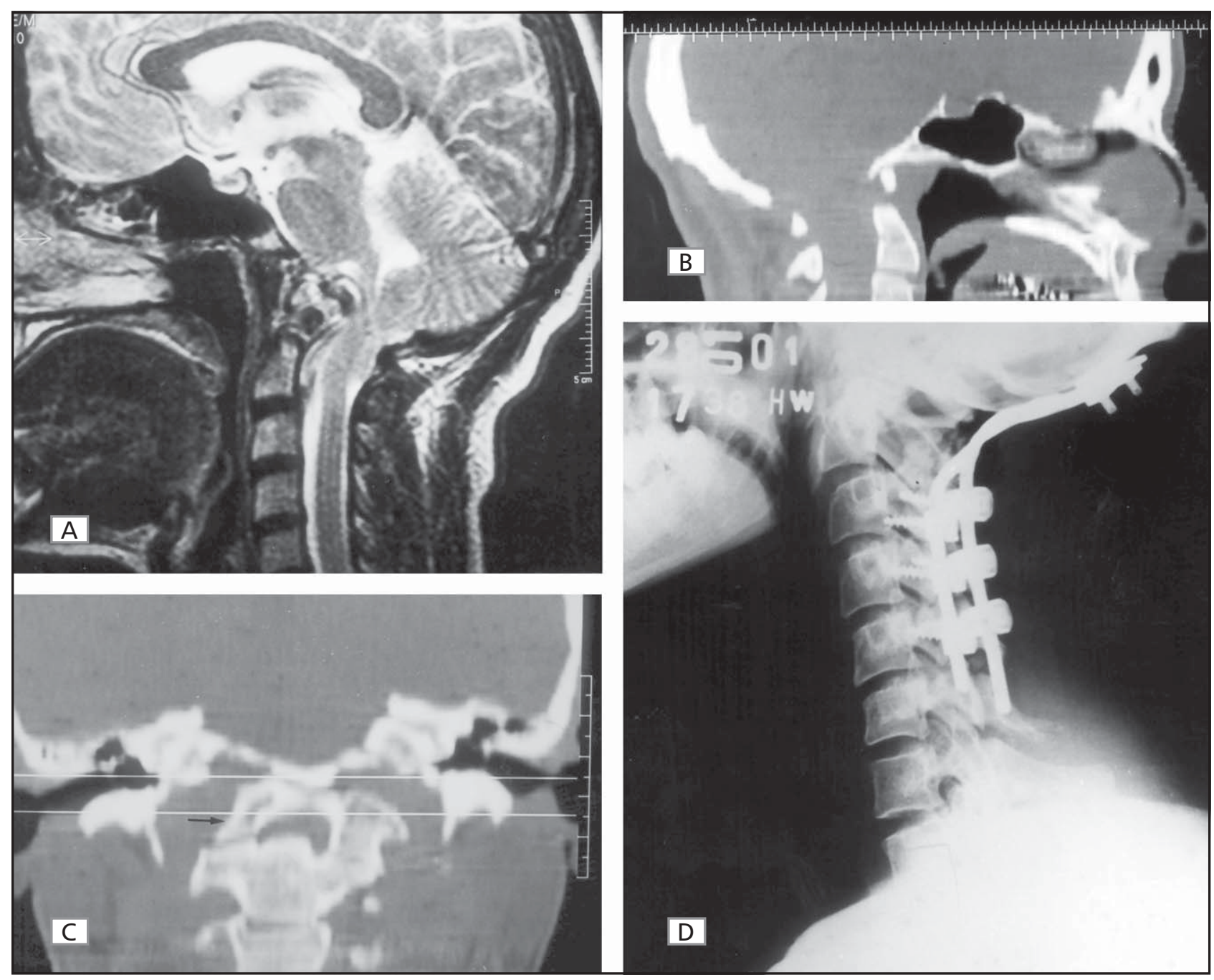

Fig 2. A) RM T2, corte sagital da transição craniocervical em paciente com impressão basilar e compressão bulbomedular; B) TC com reconstrução sagital no pós-operatório após odontoidectomia com preservação parcial do arco de C1; C) TC em corte coronal onde observa-se instabilidade $(1 C 2$; D) RX em perfil após fixação occipito-cervical.

significante mortalidade e morbidade. $O$ objetivo dos métodos de fixação é proporcionar estabilização imediata, alívio dos sintomas e correção da deformidade em um único procedimento. A fixação occípitocervical com o sistema de instrumentação "insideoutside" proposta por Pait et al. ${ }^{1}$, proporciona estabilização imediata da junção craniocervical. Os meios de fixação variam de órtese externa² a sistemas de fixação interna com osso e ou substitutos ${ }^{3}$ e instrumentos especialmente designados para este fim ${ }^{1}$. Embora os métodos tradicionais de fixação com osso associado ao uso do halo tenham sido úteis, novos sistemas de hastes ou placas fixados com parafusos, ganchos e ou arames provêem estabilidade imediata da junção craniocervical até que a artrodese se complete ${ }^{1,4-10}$.

Estudos da biomecânica da JCC demonstraram que após descompressão anterior com odontoidec- tomia, e acessos tipo transcondilar com remoção de mais de $50 \%$ do côndilo pode ocorrer instabilida$\mathrm{de}^{11-13}$. Embora a estabilização por via anterior tenha seus adeptos 4,15 , a maioria dos autores realiza o procedimento por via posterior e há consenso sobre a incorporação do côndilo occipital ao complexo atlanto-axial ${ }^{7-10}$. Isto se torna imperioso na odontoidectomia, na instabilidade por artrite reumatóide e nos tumores do corpo de C2. Dos dois casos apresentados, um fora submetido a craniectomia occipital e laminectomia de C1C2. O primeiro paciente apresentava-se com platibasia e invaginação basilar. Após descompressão anterior com odontoidectomia, na qual foi preservado o arco anterior de C1, desenvolveu instabilidade progressiva do complexo C0-C1C2. Cerca de 12 meses após a primeira cirurgia a paciente foi submetida a fixação occípito-cervical pelo método proposto. Os exames de imagem do se- 


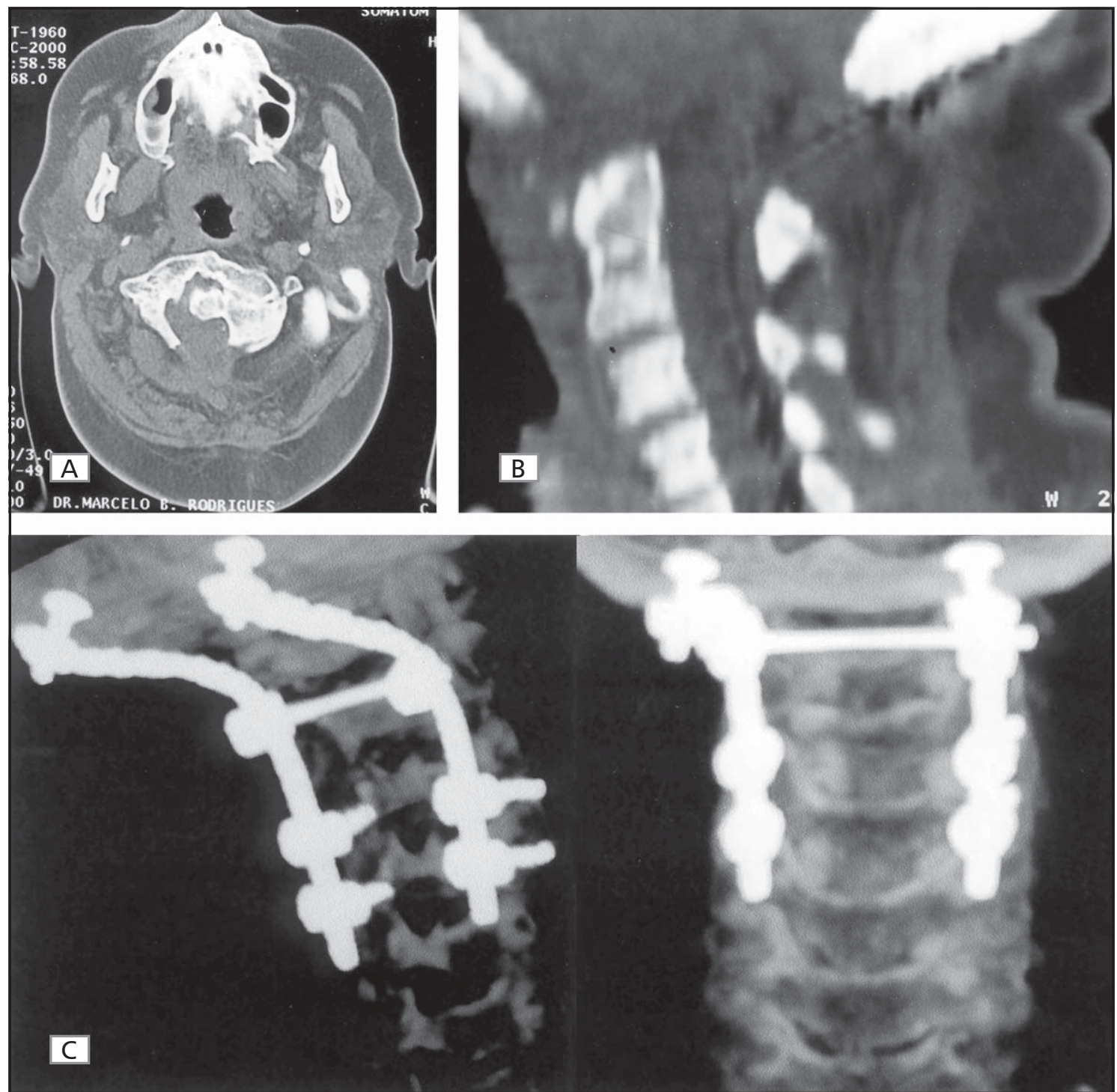

Fig 3. A) TC em corte axial da JCC mostrando luxação C1C2; B) TC com reconstrução sagital e janela óssea após odontoidectomia por via transoral;C) No pós-operatório após fixação craniocervical.

gundo paciente mostravam instabilidade do complexo C0-C1-C2 com compressão da JCC. A lesão era irredutível. A paciente foi submetida a odontoidectomia por via trans-oral e posteriormente a fixação occípito-cervical. Obviamente, os procedimentos anteriores inviabilizaram as técnicas que utilizam amarração sub-laminar e necessitam da integridade do occipital como a estabilização craniocervical com os retângulos de Luque/Hartshill ${ }^{6,8}$. Nos casos de laminectomia cervical alta, como na técnica proposta por Mori et al. ${ }^{16}, 1998$, há necessidade de estender-se a amarração sublaminar aos segmentos mais inferiores.

Embora os sistemas de estabilização com arames, como as técnicas de Rogers e Lovely\&Carls ${ }^{17}$ tenham comprovada eficácia biomecânica, estudos demonstraram a superioridade dos métodos de fixação com parafusos que provêem imobilização superior, resis- tem melhor aos testes de fadiga do que aqueles que utilizem fios metálicos e ganchos ${ }^{18}$, e o número de segmentos cervicais envolvidos é menor nas fixações com parafusos transarticulares ${ }^{19,20}$. Uma das principais vantagens da fixação occípitocervical tipo "inside-outside" é a colocação dos parafusos por dentro da haste e perpendicular ao processo articular. Este processo diminue os riscos de lesão da artéria vertebral enquanto que na fixação transarticular C1C2 a principal limitação é a proximidade da artéria. Paramore et al. ${ }^{21}, 1996$ em estudo de tomografia computadorizada de 94 pacientes, concluiram que 18 a $23 \%$ dos pacientes não são candidatos à colocação de parafusos transarticulares pelo menos em um lado por causa do trajeto da artéria dentro do buraco transverso. Madawi et al. ${ }^{22}, 1997$ em 61 pacientes submetidos à fixação $\mathrm{C} 1-\mathrm{C} 2$ com parafuso tran- 
sarticular, a maioria portadores de artrite reumatóide, tiveram cinco casos com lesão da artéria vertebral e um com paralisia transitória do nervo hipoglosso. Nos casos com lesão da artéria vertebral, somente um apresentou sintomas atribuídos à lesão desta artéria, que consistiram de acidentes isquêmicos no tronco cerebral após duas semanas da cirurgia. Eles identificaram como fatores de risco a incompleta redução antes da implantação dos parafusos, a cirurgia transoral prévia com remoção do tubérculo anterior do atlas, e falhas em identificar a correta posição da artéria vertebral junto à massa lateral e do pedículo do atlas. Estudos da "American Association of Neurological Surgeons/Congress Neurological Surgeons Section on Disorders of the Spine and Peripheral Nerves ${ }^{23 "}$ com 847 neurocirurgiões, que implantaram 2492 parafusos transarticulares em 1318 pacientes, encontraram $31(2,4 \%)$ dos pacientes com lesão da artéria vertebral e suspeita de lesão em 23 (1,7\%). Destes, somente dois exibiram déficits neurológicos e um faleceu por lesão bilateral da artéria vertebral. Outras complicações foram descritas, como laceração dural, quebra do parafuso e dormência suboccipital.

A estabilização occípito-cervical nos casos de instabilidade da articulação atlanto-axial apresenta peculiaridades por causa da complexa anatomia da região. Depende da espessura do osso occipital, da integridade óssea ao redor do forame magno, da presença ou não das lâminas, das facetas articulares e do posicionamento e do tamanho das artérias vertebrais. A fixação "inside-outside" oferece vantagens em relação aos métodos tradicionais de fixação que utiliza o parafuso de dentro para fora ou às técnicas que usem arames e cabos transfixando o occipital. Embora Heywood et al. ${ }^{24}, 1988$ não relataram complicações cerebrais com a técnica tradicional, eles evitaram penetrar com o parafuso na tábua interna. Na técnica "inside-outside" o parafuso é colocado sob visão direta com a parte achatada em contacto com a dura e o parafuso propriamente dito apontado para o cirurgião. Entretanto há necessidade de se conhecer a espessura do occipital que proporciona o suporte para a fixação do parafuso invertido.

O primeiro paciente apresentou melhora significativa da sintomatologia álgica e o segundo paciente mantém-se de pé com auxílio de órteses. $O$ controle por imagem confirma o posicionamento correto do sistema e a artrodese.

Os métodos de instrumentação devem ser de fácil manuseio, seguro e com baixo risco de provocar lesões em estruturas adjacentes. A técnica de fixação "inside-outside" preenche estes requisitos e pro- porciona estabilização craniocervical sólida até a consolidação do ciclo da artrodese.

Agradecimentos - Ao Prof. Dr. Sebastião Gusmão pela revisão do texto e ao Prof. T. G. Pait pelo estímulo a utilização da técnica.

\section{REFERÊNCIAS}

1. Pait TG, Al-Mefty O, Boop FA, Arnautovic KI, Rahman S, Ceola W. Inside-outside technique for posterior occípitocervical spine instrumentation and stabilization: preliminary results. J Neurosurg 1999;90(Suppl):1-7.

2. Pierce DS, Barr JS Jr. Fractures and dislocations at the base of the skull and upper cervical spine. In Cervical Spine Research Society. The cervical spine. 2.Ed. Philadelphia: JB Lippincot, 1989:312-324.

3. Habal MB. Differents forms of bone grafts. In Habal MB, Reddi AH (eds). Bone grafts and bone substitutes. Philadephia: Saunders; 1992:6-8.

4. Dickman CA. Biology of the spine fusion. In Dickman CA, Spetzler RF, Sonntag VKH(eds). Surgery of the craniovertebral junction. New York: Thieme, 1998:685-698.

5. Murphy M, Southwich WO. Posterior approaches and fusions. In Cervical Spine Research Society. The cervical spine, 2.Ed. Philaddelphia; Lippincot, 1989:775-791.

6. Crockard HA, Pozo JL, Ransford AO, Stevens JM, Kendall BE, Essigman WK. Transoral decompression and posterior fusion for rheumathoid atlanto-axial subluxation. J Bone Joint Surg (Br)1986;68:350-356.

7. Roy- Camille R, Saillant G, Mazel C. Internal fixation of the unstable cervical spine by a posterior osteosynthesis with plates and screws. In Cervical Spine Research Society. The cervical spine 2.Ed. Philadelphia: JB Lippincott; 1989:390-403.

8. MacKenzie AI, Utley D, Marsh HT, Bell BA. Craniocervical stabilization using Luque/ Hartshill rectangles. Neurosurgery 1990;26:32-36.

9. Grob D, Dvorack J, Panjabi, Froehlich M, Hayek J. Posterior occiptocervical fusion: a preliminary report of a new technique. Spine 1991;16:17-24

10. Grob D, Dvorak J, Panjabi MM, Antinnes JA. The role of plate and screw fixation in occípitocervical fusion in rheumatoid arthritis. Spine 1994;19:2545-2551.

11. Dickman CA, Locantro J, Fessler RG. The influence of transoral odontoid resection on stability of craniovertebral junction. J Neurosurg 1992;77:525-530.

12. Dickman CA, Crawford NR, Brantley AGU, Sonntag VKH. Biomechanical effects of transoral odontoidectomy . Neurosurgery 1995;36:1146-1153.

13. Vishteh AG, Crawford NR, Melton MS, Spetzler RF, Sonntag VKH, Dickman CA. Stability of the craniovertebral junction after unilateral occipital condyle resection: a biomechanical study. J Neurosurg (Spine1) 1999;90:91-98

14. Andrade JR, MacNab I. Anterior occipital cervical fusion using extrapharingeal approach exposure. J Bone Surg (Am) 1969;51:1621-1626.

15. Goel A, Bhatjimake M, Desai K. Basilar invagination: a study based on 190 surgically treated patients. J Neurosurg 1998;88:962-968.

16. Mori T, Matsunaga S, Sunahara N, Sakou T. 3-to11-year follow-up of occípitocervical fusion for rheumatoid arthritis. Clin Orthop Relat Res 1998;351:169-179.

17. Brasil ABV. Tratamento da instabilidade traumática da coluna cervical: avaliação biomecânica das técnicas de Rogers e de Lovely \& Carls. Tese de doutorado. São Paulo,1997.

18. Faure A, Bord E, Monteiro da Silva R, Robert R. Occípitocervical fixation with a single occipital a single clamp inverted hooks. Eur Spine J 1998;7:80-83.

19. Marcotte P, Dickman CA, Sonntag VKH, Karahallos DG, Drabier J. Posterior atlantoaxial facet screw fixation. J Neurosurg 1993;79:234-237.

20. Hurlbert JR, Crawford NR, Choi WG, Dickman C A. A biomechanical evaluation of occípitocervical instrumentation: screw compared with wire fixation. J Neurosurg (Spine1), 1999;90:84-90.

21. Paramore CG, Dickman CA, Sonntag VKH. The anatomical suitability of C1-C2 complex for screw fixation.J Neurosurg 1996; 85: 221-224.

22. Madawi AA, Casey ATH, Solanki GA, Tuite G, Veres R, Crockard HA. Radiological and anatomical evaluation of the atlantoaxial transarticular screw fixation technique. J Neurosurg 1997;86:961-968.

23. Wright MN, Lauryssen C.Vertebral artery injury in C1-C2 transarticular screw fixation: results of survey of the AANS/CNS Sections on Disorders of the Spine and Peripheral Nerves. J Neurosurg 1998;88:634-640.

24. Heywood AW, Learmonth ID, Thomas M. Internal fixation for occípitocervical fusion. J Bone Joint Surg (Br) 1988;70:708-711. 